\section{Unterbewußte Zusammenhänge in der Aetiologie der Unfall- und Renten-Neurose.}

\author{
Psychologische Betrachtungen.
}

\section{Von Siegfried Peine in Hamburg.}

Die .Neigung mancher von einem Unfall betroffener Persönlichkeiten, immer wieder auf diesem Unfall herumzureiten, ihn immer wïeder für ihre eigenen Interessen auszunützen, sozusagen den Unfall zum Angelpunkt ihrer psychischen, physischen und wirtschaftlichen Existenz zu machen, indem sie ihn bei jeder Gelegenheit in den Vordergrund ihres Interesses und des Interesses ilhrer Umgebung rücken, kann auf sehr verschiedene Art und Weise motiviert sein.

Von der bewußten Sinulation, der Vortäuschung körperlicher oder psychischer Störungen als Folgen des Unfalls, braucht hier nicht gesprochen z.11 wenden: Allerdings liegt die Versuchung nahe, bei Fehlen pathologischer Stigmata auf Simulation selbst dort zu schließen, wo eine solche im eigentlichen Sinne nicht vorliegt. Es ist ja sattsam bekannt, daB das neurotisch disponierte Individuum sehr leicht autosuggestiven Einflüssen unterliegt. Auf diesem Wege kommen vielfach auch "Krankheitsgefühle" auf, die sich bald in irgendwelche Symptome umsetzen, die der éntsprechend Veranlagte subjektiv an sich wahrnimmt, deren Feststellung sich aber der objektiven-Untersuchung entzieht. Sei es, daß der Patient - denn hier müssen wir den Betroffenen so nennen - bei anderen, ebenfalls von jenem Unfall betroffetien Personen das Auftreten solcher Störungen wahrgenommen hat, sei es, daß ihm an seinem eigenen Leibe ein - locus minoris resistentiae bekannt ist, den er nun fortgesetzt unter die psychische Lupe nimmt, bis er wirklich in seiner Einbildung eine Anomalie aufgestöbert hat, die er durch weitere Selbstbeobachtung immer mehr vor sich selbst ausbaut: in solchen Fällen handelt es sich um psychogene Motivation eines subjektiv emp fundenen Leidens, das mit Simulation nichts mehr zu tun hat und entsprechend (durch Persiasion, Suggestion usw.) behandelt werden muß, wenn sich nicht die hypochondrische Einstellung dauernd einnisten soll. Solchen Patienten Simulation vorwerfen zu wollen, wird wohl keinem psychologisch Geschulten einfallen; das Leiden, das sich schon in der fortwahihrenden, Zeit und K'raft raubenden und quälenden "Selbstbespiegelung“" als ein subjektiver Leidenszustand manifestiert, würde dadurch nur verschlimmert werden, abgesehen davon, daß auch eine antipathische Einstellung zum Arzte bzw, zu dem, der den Vorwurf erhebt, aufkommen würde, ein Umstand, der dem Schwinden der Beschwerden sicher nicht förderlich wäre.

Eine andere Art psychotraumatischer Unfallfolge, die ebenfalls keinè objektiv - nachweisbaren Schädigungen erkennen läßit; mag oft im Spiele sein, wenn eine psychisch labile Persönlichkeit an einem Unfalle beteiligt war, durch den sie selbst keine direkte Beschädigung erlitten hat, durch den aber andere Personen mehr oder wentger schwer verletzt wurden. Vermöge einer bei neurotisch disponierten Individuen besonders ausgeprägten Gefühls res on anz erleben sie sozusagen das Erlebnis eines anderen, wenn sie auch nur als Augenzeugen oder sonstwie indirekt beteiligt sind, mit, indem sie sich besonders lebhaft mit dem wirklichen oder vermeintlichen Leidenszustand jenes anderen in ihrem Vorstellungsleben beschäftigen. Dies kann bei allzu straffer Anspannung der Phantasie zu einer Art psychischer Identifikation mit dem andern führen. Diese Identifikation, dieses "Miterleben" und ,M itle ide n" fixiert sich und führt einen Dauerzustand psychischer Beunruhigung herbei, der direkt und durch Hervorrufung weiterer Störungen, die er im Gefolge hat, auch indirekt das Gesamtbild einer als eine Art Shokwirkung anzusprechenden psychotraumatischen Neurose schafft, an dem der bewußte Wille des Betroffenen keinen verantwortlichen Anteil hat:

Noch komplizierter gestalten sich die Zusammenlänge in jenen Fällen, in denen das Unterbewußtse in die treibenden Kräfte liefert. Diese verdienen daher besondere Beachtung. Auch in der Psychoanalyse; die sich ja eingehend mit der Psychologie des Unterbewußtseins befaßt, "erscheinen diese Zusammenhänge noch nicht scharf genutg herausgestellt. Ihnen wollen wir uns in Folgendem zuwenden.

Es mag in jenen Fällen eine sehr energische Abzielung auf die Erlangung materieller Vorteile aus dem Unfall zu erkennell sein. Das Streben des vom Unfall Betroffenen richtet sich mit starker Affektbetonung auf die Erzielurng einer Rente, und zwar einer möglichst hohen. Es entwickelt sich das Bild dessen, der pathologische Erscheinungen provoziert, nur um materiellen Gewinn aus dem Unfalle heimzubringen. Und doch ist die Kapitalerlangung solcher Individuen gar nicht das eigentliche Ziel. Sie ist nur ein vorgeschobenes Ziel, während das eigentliche ihnen selbst meist unbewußt oder doch so tief unter der Schwelle des Bewußtseins verankert ist, daß sie es nicht ohne weiteres erkennèn können, sondern bei seiner Verfolgung unter einem inneren Zwange handeln. Inssbesondere, wenn es sich um bemittelte Personen handeit, die mit heftiger Giebärde und unverhältnismäßig. s atker Anspannung ihrer Energie auf Kapital bzw. Rente drängen, nuß der Verdacht aufsteigen, daB die Affektivität ihre Kräfte weniger aus der materiellem Sphäre als vielmehr aus einer unterbewụßten Sphärę bezieht, der das Materielle, das Geldliche an sich nicht Gegenstand ihres Hauptinteresses ist. Bekanntlich findet sich beim Neurotiker die Tendenz, ials krank gelten und sich auf diese Weise das Mitleid und die Fürsorge seiner Umgebung sichern zu wollen. Mag es sich um die angeborene oder erworbene Minderwertigkeit irgendeines Organes handeln, die ein Schwächegefühl erzeugt (Adlèr), oder mag ein verdrängtes, nicht abreagiertes und nun aus ${ }^{3}$ der Unterbewuß theit heraufwirkendes psychisches Trauma das Individuum beunruhigen (Freud), jedenfalls neigt der Neurotiker dazu, seine Umgebung, seine Angehörigen in seinen Dienst zu stellen. Einmal benützt er seine Umgebung als Stütze, mittels derer er sein Schwächegefühl auszugleichen trachtet, ein andermal sucht er sich, dem vermindert Liebestähigen, wenigstens eine Art Liebesersatz zu verschaffen, in dem er seine Umgebung zu dauernder Betreuung seiner selbst an sich kettet und allerhand "Liebesdienste“ an sich ausüben läßt. Wer glaubt, wenig ,ta us Liebe“ empfangen zu können, etwa weil er selbst sich nicht potent zu voller Liebesbetätigung fühlt, der wird es gern haben, wenn man •ihm recht viel ,z u Liebe“* tut. Und er wird alles daran setzen, sich in eine Positión zu bringen, die ihm ein Anrecht auf Bemitleidet- und Beruhigtwerden und auf Liebesgewinn einträgt. Der Neurotiker wird von seiner Umgebung of nicht ernst genommen. $\mathrm{Er}$ hat daher ein Interesse daran, sofern sich eine Gelegenheit dazu bietet, sich seine Schwäche, sein Recht auf Liebesempfang amtlich anerkennen und bestätigen $z u$ lassen. Ein erlittener Unfall stellt sich als eine geeignete Gelegenheit zur Erlangung eines solchen $S$ chwächeausweises oder "Liebespasses" dar. Jetzt "darf" er krank geworden sein, jetzt "ist ein Anla $\beta$ zum ,wirkliche.1" Krankwerden vorhanden, er kann sozusagen auf anständige Art in die Neurose flüchten, während man ihm früher mangels einer offenkundigen Veranlassung seine Beschwerden nicht recht geglaubt hat. Nicht bewußt, denn das wäre wieder Simulation, sonderu unbewußt raunt er sich $z u$ : "Die Gelegenheit is günstig, jetzt heißt's 'sic nützen, aber auch gleich so, daB einem das Kranksein gehörig be gla ubigt wird und man da uernde n A nspruch auf Mitleid und Liebe erwirbt. $\leftrightarrows$ Was aber ist eine bessere. Beweissicherung für das Kranksein als die Rente, die immer wiederkehrt und immer aufs neue proklamiert: dieser da is ein bemitleidenswerter Mensch, der unverschuldet (auch zu beachten bei Vorliegen etwaiger eigener Schuldkomplexe, wovon weite unten noch die Rede sein wird) und $z$ ufällig (zugleich eine Sicherung gegen das mißliche Momerf, als geborener Schwächling zu gelten) ins Kranksein hineingeraten ist. Die affektive Verfechtung des Rentenanspruchs ist in solchen Fällen als der symbolische Ausdruck des Anspruchs auf Mitleid und seelische Unterstützung ıaufzufassen, Der Neurotiker will aus dem Un fall Kapital schlagen, aber es ist thm .weniger um das Kapital selbst zu tun als um die psychologischen Wirkungender Kapitalserlangung auf seine Umgebung. Das Kapital besteht für ihn nicht eigentlich in der Rente, sondern im wesentlichen in der Anerkennung als Mitleid-, Liebes- und „Unterstützungsbedürftiger“. Sein Kampf um Rente ist also in übertragener Bedeutung zu verstehen.

Liegt Anlaß zur Annahme eines solchen Zusammenhanges vor, dann wird sich die Psychoanalyse als zweckdienlich erweisen. Es ergibt sich schon aus obiger Darstellung, daß dabei weder eine einseitige Fahndung anf sexuelle Kindheitstraumen noch eine solche allein auf Minderwertigkeits- oder Schuldkomplexe in Frage kommt sondern es muß dabei aus allen "Richtungen" der Psychoanalyse geschöpft werden, da bald diese, bäld jene ursächliche Verstrickung den Kern der Neurose bilden kann. Daß. der Boden für das Aufkommen einer solchen Neurose günstig sein, also eine entsprechende Disposition vorliegen muß, braucht nicht erst gesagt zu werden das ist aber hier auch unerheblich, da wir eben nur von solchen Individuen reden, bei denen eine dispositionelle Eignung zur unterschwellig verankerten Neurose besteht. Ob eine solche Eignung bzw. ein dispositionelles Entgegenkommen zur Verdrängung vorliegt, läßt sich wohl erst im Verlaufe der Psychoanalyse selbst erkennen.

Es ist schon kurz auf Schuldkomplexe hingewiesen worden, die den Neurotiker beherrscherr können. Im Rahmen der vorliegenden Abhandlung interessiert uns dieses. Moment insofern, als es ätiologische Bedeutung fïir die Entwicklung einer Unfall- und RentenNeurose gewinnen kann, wenn der Neurotiker von allerhand Schuldvorstellungen gequält wird -(z. B. wegen realisierter oder anch nur stiller aber affektiver Auflehnung gegen den Willen des Vaters oder wegen irgendwelcher erotischer Abwegigkeiten) und er nun in seiner Vorstellung lebendige Schuld gelten und Crux befreien kann. (Ueber die "A ngst als Strafe" haben sich Freud und Kaplan des näheren verbłeitet.) Bestraft und gesühnt ist aber die. Schuld nur dann, wenn er einen bleibenden Denkzettel von jenem Unfall aufweisen kann, und ein solcher Denkzettel ist wieder die Rente, die er darum mit starker Affektivität zu erlangen trachtet, indem er zum Ausgleich seines Schuldkon tos irgendein (anderes, nach außen hin auffälligeres) netrotisches Symptom, ohne daß ihm der Hergang bewußt wird, produziert. Er ist nun vor seinem Ich einmal von jener „Schuld“ entsühnt, denn 'er wertet ja das Unfallerrlebnis und die im Anschlusse daran von seinem Unterbewußtsein arrangierte Neurose als Läuternngsproze $B$. Dadurch ist er aber nun in Zukunft wirklich , unverschuldef krank, weil eben das Schuldmoment in der Aetiologie seiner neu rotischen Beschwerden. infolge der eingetretenen Verschiebung für 
ihn erledigt ist. Inden der Neurotiker die Krankleitsursache auf die unschuldige Zufälligkeit des Unfalls verschiebt, sichert er sich gegen den Selbstvorwurf der Schuld. Er kann sich jetzt dem "Genusse" seiner Neurose, wern man so sagen darf, freier und "unbefangener lingeben, und das ist zugleich das Signal für die neurotischen Symptome, sich freier zu entfalten, als ihm dies vor dếm Unfall gestattet war. Das Unterbew uBtsein hat auch hier wieder ein lebhaftes Interesse daran, jenes, "neurotische Arrangem e $\mathbf{n}^{\text {t" }}$ durch Erzielung einer Dauerrente als Unfallfolge dokumentiert zu sehen, weil dadurch das Persönlichkeitsgefühl des Neurotikers eine Steigerung erfährt. Auch diesen Zusammenhängen gegenüber erscheint psychoanalytische Aufhellung am Platze; jedenfalls wird sie zweckfördernd in die Behandlung mit einzubeziehen sein. 